Cite this: Phys. Chem. Chem. Phys., 2012, 14, 914-919

\title{
The existence region and composition of a polymer-induced liquid precursor phase for DL-glutamic acid crystals $\dagger$
}

\author{
Yuan Jiang, $\ddagger^{a}$ Laurie Gower, ${ }^{b}$ Dirk Volkmer ${ }^{c}$ and Helmut Cölfen $\ddagger^{* a}$ \\ Received 9th June 2011, Accepted 3rd November 2011 \\ DOI: $10.1039 / \mathbf{c 1 c p 2 1 8 6 2 j}$ \\ The existence region of a polymer-induced liquid precursor (PILP) phase for crystals of an \\ organic compound (DL-glutamic acid, Glu) was determined for the first time in the phase diagram \\ of the Glu-polyethyleneimine-water-ethanol system. The existence region and the amount of \\ PILP phase relative to the thermodynamically stable crystal phase were very small. Other phases \\ detected in the phase diagram were coacervates, homogenous mixtures, and crystals obtained via \\ a clear solution. The PILP phase is rich in the polymeric additive, which helps to explain the long \\ induction period of PILP before crystallization occurs. Volume measurements indicated that its \\ amount is $\ll 1 \mathrm{vol} \%$, showing that this precursor phase is only a minor component.
}

\section{Introduction}

Biomineralization and biomimetic mineralization reactions challenge the classical layer-by-layer crystal growth model. ${ }^{1}$ Nonclassical particle- and precursor-based mechanisms have been revealed. ${ }^{2}$ Precursors can exist as amorphous ${ }^{3,4}$ or crystalline $^{5-7}$ nanoparticles as well as condensed liquid phases. ${ }^{8-13}$ Especially important are the amorphous precursor phases found to be involved in biomineralization. ${ }^{14,15}$ Amorphous nanoparticle precursors are transient phases, so it is difficult to separate them from reaction systems for compositional and structural studies. In contrast, liquid precursors prepared under the same conditions are reported to grow up to micrometre-sized droplets and they can be stabilized for some minutes to hours. ${ }^{12,16,17}$ Therefore, it is possible to observe the existence of liquid precursors and their transformation into crystalline structures by simple optical microscopy.

Among liquid precursors, the concept of a "polymer-induced liquid precursor" (PILP) is of special interest because it potentially provides a general way to control phase transformation processes for a broad range of crystalline materials, some of

\footnotetext{
${ }^{a}$ Max Planck Institute of Colloids and Interfaces, Colloid Chemistry, Am Mühlenberg, D-14476 Golm, Germany

${ }^{b}$ Materials Science \& Engineering, University of Florida, Gainesville, FL 32611, USA

${ }^{c}$ Institute of Physics, Chair of Solid State \& Materials Chemistry, Augsburg University, D-86159 Augsburg, Germany

$\dagger$ Electronic supplementary information (ESI) available: The microscopy images of PILP, coacervate, and crystals formed via the PILP phase; the titration measurements for the precise determination of the boundaries for the PILP formation; the ultracentrifugation measurements for the PILP as well as coacervate phases; the NMR results for the coacervates. See DOI: $10.1039 / \mathrm{c} 1 \mathrm{cp} 21862 \mathrm{j}$

$\ddagger$ Present address: Physical Chemistry, University of Konstanz, Universitätsstr. 10, D-78457 Konstanz, Germany. E-mail: helmut.coelfen@unikonstanz.de; Fax: + 49 (0)7531-88-3139; Tel.: + 49 (0)7531-88-4063
}

which have significant medical or technological applications. The concept of PILP was initially put forward by Gower et al. for model systems based on $\mathrm{CaCO}_{3} \cdot{ }^{9-11}$ The crystallization system can pass through a PILP phase during the crystallization of $\mathrm{CaCO}_{3}$ in the presence of $\mu \mathrm{g} \mathrm{mL}^{-1}$ amounts of a polyacid such as poly(acrylic acid) or poly(aspartic acid). The fluidity of the PILP phase allows it to be shaped into various nonequilibrium crystalline structures. ${ }^{11,18,19}$ For example, Gehrke et al. reported the retrosynthesis of nacre via the PILP route. ${ }^{18}$ The authors directly used the insoluble organic nacre-like matrix as a confined reaction environment, and PILP could flow inside and grow biomimetic nacre structures in situ. ${ }^{20}$ In addition, Gower et al. demonstrated that intrafibrillar mineralization of collagen can be achieved with either calcium carbonate or calcium phosphate through infiltration of the interstices of the collagen fibrils with a precursor phase. ${ }^{21-23}$ Very recently, Sommerdijk et al. presented high resolution cryo-transmission electron microscopy work on this process, which revealed that prenucleation clusters ${ }^{24,25}$ and an amorphous precursor were involved. ${ }^{26}$ Indeed, the amorphous phase has been proven relevant in the formation of biominerals. ${ }^{4,14,15}$ Although it is not currently possible to determine if the PILP route is responsible for the amorphous phase, such a concept is advantageous for explaining the formation of crystals with complex forms like biominerals. In addition, it has been applied for various morphosyntheses of biomimetic materials, such as $\mathrm{CaCO}_{3}{ }^{11}$ $\mathrm{Ca}_{5}\left(\mathrm{PO}_{4}\right)_{3}(\mathrm{OH}),{ }^{22} \mathrm{SrCO}_{3},{ }^{27}$ and $\mathrm{BaCO}_{3}{ }^{27,28}$ into powders, fibers, micromolded structures, ${ }^{29,30}$ and thin tablets ${ }^{31}$ and films, ${ }^{10}$ and has a great potential for complex morphosynthesis applications.

The determination of PILP composition and possible structure is of primary importance for understanding crystallization via the PILP route. Prior PILP research has mainly focused on inorganic mineralization systems. For example, Dai et al. reported that the mineral content within the PILP phase was 
increasing with time in a $\mathrm{CaCO}_{3}$-poly(aspartic acid) reaction system. ${ }^{17}$ In addition, the molar ratio of $\mathrm{Ca}^{2+} / \mathrm{CO}_{3}{ }^{2-}$ is larger than one in the early stages of the $\mathrm{CaCO}_{3}$ PILP phase, but the value approaches stoichiometry with time. Therefore, the PILP transformation into crystalline phases is suggested to be a continuous process in mineralizing systems. Importantly, the existence of polymeric additives in the PILP phase can stabilize the reacting systems against immediate crystallization, thereby providing a means to stabilize the nonequilibrium morphology of the amorphous precursor as it crystallizes. ${ }^{11}$ Using synchrotron in situ X-ray reflectivity, DiMasi and coworkers showed that the existence of soluble poly(acrylic acid)-sodium salt could extend the lifetime of amorphous or liquid-like precursors. ${ }^{32,33}$ However, it is not yet clear over which range of compositions they are formed. Another barrier in the understanding of the PILP formation by using a reacting mineralization system lies in the difficulty to distinguish PILP from metal cationpolyelectrolyte complexes. For example, metal cations can become complexed by a polyanion, leading to dissolved complexes. Another important issue is the polymer content. The existence of an equal amount of counter-charged charged polymers and the crystallizing molecules can stabilize the molecules from crystallization. Instead, a thermodynamically stable molecule-polymer coacervate phase is formed in equilibrium with a supernatant phase. On the other hand, enough polymer must be present to interact with the metal cations to ensure that all crystalline materials are formed via the PILP route; otherwise, crystallization occurs without passing through the PILP phase, leading to crystal side products (such as aggregates of faceted crystals).

To find an appropriate system for better understanding of PILP formation, the Cölfen group has extended the method to crystallization of organic molecules with oppositely charged polyelectrolytes. ${ }^{12,34}$ For example, Wohlrab et al. reported that a PILP phase could be stabilized for hours or days before the precipitation occurred. ${ }^{12}$ However, it is still unclear over which compositional range in the phase diagram PILP phases can be found, which is important information if these phases shall be produced in larger quantities.

The work presented herein is a study of the Glu-PEI (Glu: DL-glutamic acid; PEI: branched polyethylene imine with average $M_{\mathrm{w}}=800 \mathrm{~g} \mathrm{~mol}^{-1}$ ) PILP system over a large range of compositions to determine the phase behavior as well as the existence region of the unstable PILP phase. In addition, the composition and amount of organic PILPs were determined using NMR spectroscopy and analytical ultracentrifugation, respectively.

\section{Experimental section}

DL-Glutamic acid (Glu) monohydrate and branched polyethyleneimine (PEI; average $M_{\mathrm{w}}=800 \mathrm{~g} \mathrm{~mol}^{-1}$ ) were purchased from Aldrich and used without further purification. Water purified with a Milli-Q system was used for the preparation of aqueous solutions. The reagent grade absolute ethanol $(\mathrm{EtOH} ; 200$ proof, 99.5\%) was purchased from Acros Organics.

A series of aqueous solutions containing various concentrations of Glu and PEI were prepared. Then, aqueous solutions were mixed with EtOH at a certain volume ratio. The phase boundary between the precipitation via the PILP phase and that via the clear solution was determined by a titration process. EtOH was titrated into a Glu-PEI aqueous solution with

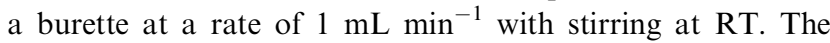
turbidity could be observed directly by eyes. The first boundary point was recorded when the mixture immediately turned turbid and the turbidity persisted over $1 \mathrm{~min}$; while the second one was determined when the turbidity disappeared.

The PILP phase was separated from the supernatant phase by centrifugation (7000 rpm; $5 \mathrm{~min}$ ). After the complete evaporation of solvent, the remaining chemicals were dissolved in $\mathrm{D}_{2} \mathrm{O}$ for NMR measurements. The ${ }^{1} \mathrm{H}$ NMR measurements were carried out on a Bruker-DMX 400 instrument at $25{ }^{\circ} \mathrm{C}$. The molar ratio of Glu to the repeat unit of EI $\left(n_{\mathrm{Glu}} / n_{\mathrm{EI}}\right)$ in each phase was determined as follows. First, a series of Glu-PEI aqueous solutions with various values of $n_{\mathrm{Glu}} / n_{\mathrm{EI}}$ were measured by ${ }^{1} \mathrm{H}$ NMR spectroscopy to obtain a standard calibration curve (Fig. S6A, ESI $\dagger$ ). Next, the peak ratio of broad hydrogen peaks on hydrocarbon groups of PEI and $\gamma$-H peaks of Glu was measured and calculated (Fig. S6B, ESI $\dagger$ ). Finally, the values of $n_{\mathrm{Glu}} / n_{\mathrm{EI}}$ in each phase were determined by comparing the peak ratios with the standard curve.

Ultracentrifugation measurements were performed on an XLI ultracentrifuge (Beckman Coulter) (PaloAlto) equipped with Rayleigh interference optics. A $340 \mu \mathrm{L}$ sample of water was loaded into the left sample sector as the reference. In the right sample sector, a certain volume of $\mathrm{EtOH}$ was charged followed by $30 \mu \mathrm{L}$ FC-43 (an immiscible oil with high density, which will stay at the bottom during centrifugation for better data reading of the bottom position). Then, a quantity of Glu-PEI aqueous solution was charged into the right sample sector to mix with the EtOH in this sector. The sealed cells were run for $1 \mathrm{~h}$ at $10000 \mathrm{rpm}$ before data reading to ensure coalescence of the PILP droplets and the formation of a two-phase system. From the sharp phase boundary positions detected by the optical detection system and the known geometry and dimensions of the cell, the volumes of the coexisting phases could be determined using the formula $V=\left(r_{\mathrm{b}}^{2}-r_{\mathrm{m}}{ }^{2}\right) \times h \times$ $\pi \times \varphi / 360^{\circ}$, where $V$ is the volume measured, $\varphi$ is the sector angle of the ultracentrifuge cell, $r_{\mathrm{b}}$ and $r_{\mathrm{m}}$ are radii of the top and the bottom of the layer of interest from the rotor centre, respectively, and $h$ is the thickness of the cell. A Leica DMRB microscope with polarizers was used for the characterization of coacervates and precipitates.

\section{Results and discussion}

The standard quaternary system contains Glu, PEI, water, and ethanol (EtOH). Glu is a zwitterion carrying two carboxyl groups and one amine group $\left(\mathrm{pKa}_{1}=2.2, \mathrm{pKa} \mathrm{a}_{2}=4.2\right.$, $\mathrm{pKa}_{3}=9.7, \mathrm{p} I$ (isoelectric point) $\left.=3.2\right)$. PEI $(\mathrm{pKa}=10.2)$ is rich in primary, secondary and tertiary amine groups (only primary and secondary amine groups interact with carboxyl groups on Glu) (Fig. 1). Therefore, oppositely charged Glu and PEI can interact with each other mainly via electrostatic interactions in aqueous solution within a wide $\mathrm{pH}$ range between $\sim 2.2$ and $\sim 10.2$. The $\mathrm{pH}$ value of the initial aqueous solution investigated was between 3.2 and $\sim 10$, and it depended on the value of $n_{\mathrm{Glu}} / n_{\mathrm{EI}}$ as well as the [Glu] (or [PEI]). The experiments were performed by mixing the Glu-PEI aqueous 


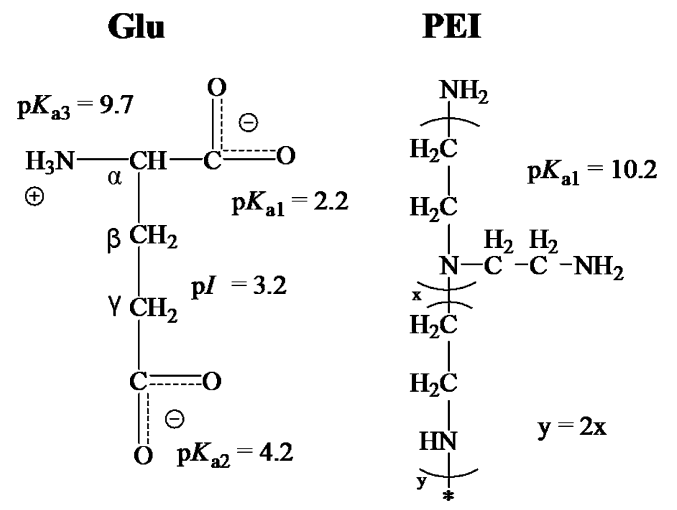

Fig. 1 The molecular structures of Glu and PEI, along with their associated $\mathrm{p} K_{\mathrm{a}}$ values.

solution with EtOH, which is a non-solvent for Glu (At $25^{\circ} \mathrm{C}$, Glu solubility is $0.11 \mathrm{~mol} \%$ in pure water and $8.3 \times 10^{-4} \mathrm{~mol} \%$ in EtOH). ${ }^{35}$ Without PEI, Glu is known to precipitate into single crystalline sheets directly from the water-EtOH mixture. ${ }^{36}$ However, the existence of PEI can protect Glu from immediate precipitation and different Glu/PEI ratios can result in different phase behaviors of the quaternary system.

The first part of this study focused on the phase behavior of the Glu-PEI-water-EtOH system. The aim was to reveal the proper compositions for Glu precipitation via the PILP route. First, we found that the molar ratio of Glu to EI-the repeating structural unit of $\mathrm{PEI}-\left(n_{\mathrm{Glu}} / n_{\mathrm{EI}}\right)$ was the most important parameter for determining the phase behavior of the quaternary system herein. By decreasing $n_{\mathrm{Glu}} / n_{\mathrm{EI}}$, setting $V_{\mathrm{EtOH}} / V_{\text {water }}=9$, we observed four possibilities of phase behaviors in sequence: precipitation from a clear solution, precipitation via the PILP route, coacervate formation, and the formation of dissolved complexes (Fig. 2). Both PILPs and coacervates are liquid-like droplets with a wide size distribution from several microns to hundreds of microns. Both of them can cause turbidity in newly-formed quaternary mixtures. They could not be distinguished directly from optical microscopy (OM) images, but from the observation of the final products. Crystalline microspheres were obtained via the PILP route (Fig. 2B, and Fig. S1, ESI †: (A) PILP droplets, (B and C) crystalline microspheres), while coacervate droplets coalesced and formed a continuous liquid phase (Fig. S1D, ESI $\dagger$ ). Coacervates could be obtained via phase separation from a homogenous Glu-PEI aqueous solution by addition of EtOH as well as from mixing Glu aqueous solution with PEI alcoholic solution. The principle of path independence of reaching the stable state hints at the thermodynamic stability of the coacervates. It must be underlined that a PILP phase is not thermodynamically stable since it leads to final crystals as thermodynamically stable products. It is only a kinetic precursor phase.

To visualize the phase behavior, two series of pseudo ternary phase diagrams were created by keeping $n_{\mathrm{Glu}} / n_{\mathrm{EI}}$ or $V_{\text {EtOH}} / V_{\text {water }}$ constant. Herein, the EI concentration was used as the axis for better understanding of the interactions between Glu and each repeating structural unit in PEI. First, a representative pseudo ternary phase diagram was drawn (Fig. 3A; $\left.V_{\mathrm{EtOH}} / V_{\text {water }}=9\right)$. The boundary between the area for the formation of homogenous complexes and that for coacervation was determined by collecting as many as possible data points near the boundary area. The formation of a homogeneous mixture, where the Glu-PEI complexes were dissolved in water-EtOH mixtures, was detected for $n_{\mathrm{Glu}} / n_{\mathrm{EI}}$ lower than $\sim 0.1-0.05$, and the exact value varies with [Glu] and [EI]. If [Glu] and [EI] were comparable in the water-EtOH mixtures, a coacervation process occurred. Most Glu-PEI complexes were accumulated in the dense liquid phase, in equilibrium with the dilute supernatant phase. Below the coacervation area, Glu crystals were in equilibrium with a saturated Glu solution phase in a water-EtOH-PEI mixture. Precipitation via the PILP phase from the quaternary mixture could only be detected in a range of low [Glu] and relatively large values of $n_{\mathrm{Glu}} / n_{\mathrm{EI}}$. For example, most precipitation data fell into the range of [Glu] between 0 and $0.15 \mathrm{~mol} \%$, as shown in Fig. 3B-a zoomed-in image of Fig. 3A. The phase boundary between coacervation and precipitation via the PILP route is clearly seen when $n_{\mathrm{Glu}} / n_{\mathrm{EI}}$ is between 1 and 0.5 . In addition, the phase boundary between precipitation via the PILP phase and that from a clear solution can be seen in Fig. 3B. In the precipitation area, precipitation proceeded directly via the PILP route when [Glu] was higher than $\sim 0.015 \mathrm{~mol} \%$ and the value of $n_{\mathrm{Glu}} / n_{\mathrm{EI}}$ was lower than 12 . Otherwise, pure single crystalline precipitates were obtained. To summarize, the boundaries between different phase behaviors are clearly seen in Fig. 3A and B. The area for Glu precipitation via

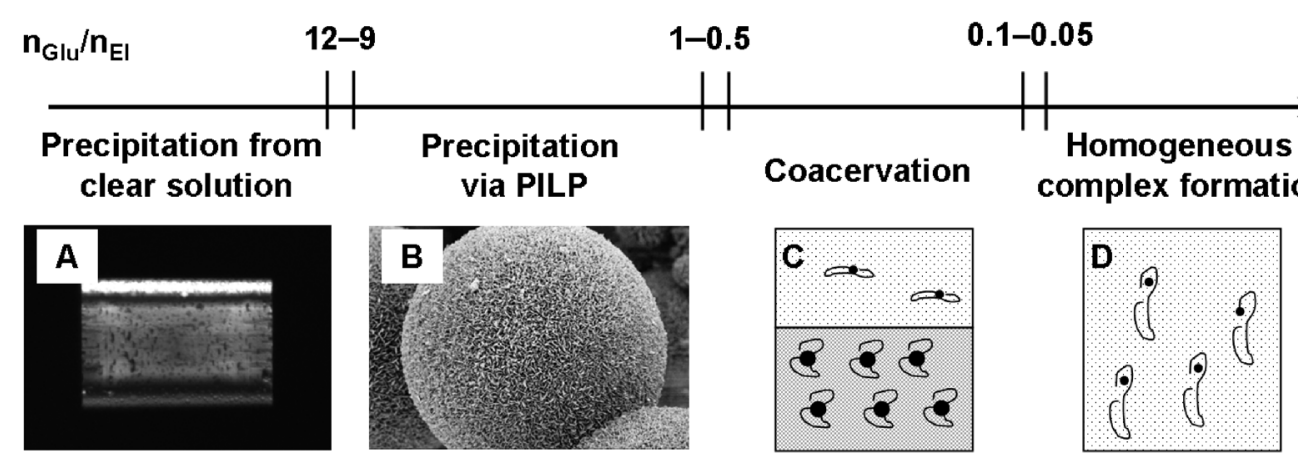

Fig. 2 The sequence indicates the relationship between the values of $n_{\mathrm{Glu}} / n_{\mathrm{EI}}$ and the phase behavior in a quaternary Glu-PEI-water-EtOH system. $V_{\text {EtOH }} / V_{\text {water }}=9,[\mathrm{Glu}]=0.12 \mathrm{~mol} \%$ in the initial Glu-PEI aqueous solution with variable [PEI]. Single crystalline precipitates were obtained via the precipitation from a clear solution (A), while microspheres were precipitated via the PILP route (B). A coacervation process caused the formation of a coacervate phase rich in Glu-PEI complexes (the possible isolated Glu and/or PEI molecules are not shown). Glu-PEI complexes were soluble and homogeneously distributed in a quaternary mixture when the value of $n_{\mathrm{Glu}} / n_{\mathrm{EI}}$ was lower than $\sim 0.1$. 

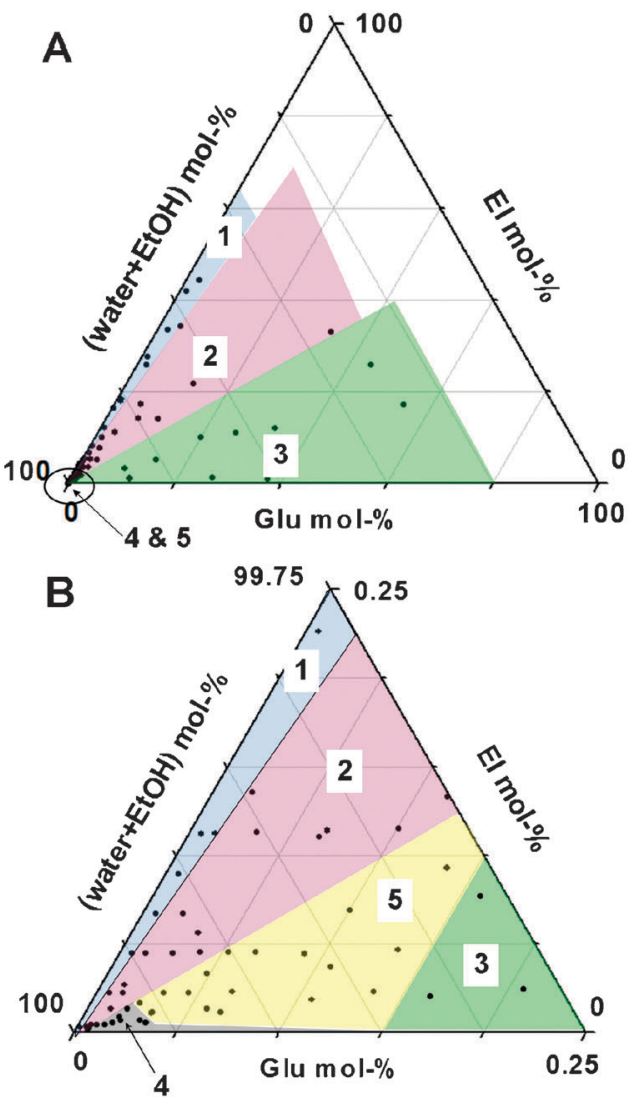

Fig. 3 Two images show the phase behavior of the Glu-PEI-waterEtOH system $\left(V_{\mathrm{EtOH}} / V_{\text {water }}=9\right)$. The three axes are Glu, ethylene imine (EI) repeat unit, and (water $+\mathrm{EtOH}$ ) $\mathrm{mol} \%$. Image (A) is a full pseudo ternary phase diagram, indicating the areas for the formation of homogeneous complexes (zone 1), coacervation (zone 2), and the coexistence of Glu crystals and saturated Glu solution phase in a water-EtOH-PEI mixture (zone 3). The phase behavior in areas with high [Glu] or [PEI] was not measured because the high solute content caused difficulties in thorough mixing of the components. Image (B) shows the circled area in Image (A), indicating the area for the precipitation via a clear solution (zone 4) as well as that for the precipitation via the PILP route (zone 5).

the PILP route is very small. In practice, the range for precipitation via the PILP route can be further enlarged towards a higher [Glu] range over $0.15 \mathrm{~mol} \%$ by increasing the solubility of Glu by a temperature increase or switching the $\mathrm{pH}$ value of the aqueous solution far from the $\mathrm{p} I$ of Glu. ${ }^{37}$

To clarify the exact phase boundaries between precipitations via a clear solution and via the PILP route, the phase behavior of the quaternary system was investigated by keeping $n_{\mathrm{Glu}} / n_{\mathrm{EI}}$ at $1.5,3.1$, and 6.2 . The method was performed by titrating a Glu-PEI aqueous solution with $\mathrm{EtOH}$, and the turbidity of the mixture was judged directly by eye. At the beginning of the titration, the quaternary mixture was clear. At a certain point, the mixture immediately turned turbid. The existence of PILP was further confirmed by optical microscopy (droplets and subsequent crystallization, Fig. S1A-C, ESI $\dagger$ ). The value of $V_{\mathrm{EtOH}} / V_{\text {water }}$ at this point was calculated $\left(V_{\mathrm{EtOH}} / V_{\text {water }}^{\prime}\right)$. The turbidity decreased thereafter upon further EtOH addition, and it finally disappeared by continuing addition of EtOH $\left(V_{\mathrm{EtOH}} / V_{\text {water }}^{\prime \prime}\right)$. Therefore, precipitation via the
PILP route proceeded in the range between $V_{\mathrm{EtOH}} / V_{\text {water }}^{\prime}$ and $V_{\mathrm{EtOH}} / V_{\text {water }}^{\prime \prime}$, while precipitation via a clear solution occurred outside of this range. The results from the three series of $n_{\mathrm{Glu}} / n_{\mathrm{EI}}$ values are shown in Table $\mathrm{S} 1 \mathrm{~A}-\mathrm{C}(\mathrm{ESI} \dagger)$.

To analyze the data in Table S1 (ESI $\dagger$ ), we first considered the relationship between the range of $V_{\mathrm{EtOH}} / V_{\text {water }}$ and [Glu] $\left(n_{\mathrm{Glu}} / n_{\mathrm{EI}}\right.$ was constant) for the precipitation via the PILP route. We observed that the turbidity range was decreasing with the decrease of [Glu] (Table S1A-C, ESI $\dagger$ ). This phenomenon proves again that a high value of $[\mathrm{Glu}]$ is beneficial for precipitation via the PILP route within the existence region of PILP. Furthermore, the relationship between the range of $V_{\mathrm{EtOH}} / V_{\text {water }}$ and $n_{\mathrm{Glu}} / n_{\mathrm{EI}}$ for the precipitation via the PILP route was investigated. The gaps between $V_{\mathrm{EtOH}} / V_{\text {water }}^{\prime}$ and $V_{\mathrm{EtOH}} / V_{\text {water }}^{\prime \prime}$ for the precipitation via the PILP route decrease with the increase of $n_{\mathrm{Glu}} / n_{\mathrm{EI}}$, as shown in Table S1A-C (ESI $\dagger$ ). For example, the values of $V_{\mathrm{EtOH}} / V_{\text {water }}^{\prime}$ and $V_{\mathrm{EtOH}} / V_{\text {water }}^{\prime \prime}$ are 2.2 and 38 , respectively, in the quaternary mixture obtained by using a concentrated Glu-PEI aqueous solution $\left(n_{\mathrm{Glu}} / n_{\mathrm{EI}}=3.1\right.$; $[\mathrm{Glu}]=0.12 \mathrm{~mol} \%$ ), while the corresponding values are 2.4 and 19 for another Glu-PEI aqueous solution $\left(n_{\mathrm{Glu}} / n_{\mathrm{EI}}=6.2\right.$; $[\mathrm{Glu}]=0.12 \mathrm{~mol} \%)($ Table S1B and $\mathrm{C}, \mathrm{ESI} \dagger)$.

From the combination of the results from Fig. 3 and Table S1 $(\mathrm{ESI} \dagger)$, we draw the conclusion that precipitation via the PILP route is preferred when using a nearly saturated Glu-PEI aqueous solution with a value of $n_{\mathrm{Glu}} / n_{\mathrm{EI}}$ between $\sim 1$ and $\sim 10$. The PILP phase can be generated when the above aqueous solution is mixed with $\mathrm{EtOH}$ with the value of $V_{\mathrm{EtOH}} / V_{\text {water }}$ between $\sim 5$ and $\sim 20$. The exact value is variable, depending on the exact composition of the quaternary system.

After determining the range in the pseudo ternary phase diagram for the precipitation via the PILP route, the volume and composition of the PILP phase were determined by using analytical ultracentrifugation (AUC) and NMR, respectively. AUC was applied to detect the volume of the PILP phase by coalescing the droplets in the centrifugal field and measuring the volumes of the PILP phase as well as the whole volume of the mixture (details available in the Experimental section). Thereby, the volume fraction of PILP was determined. The effects of [Glu], $n_{\mathrm{Glu}} / n_{\mathrm{EI}}$, and $V_{\mathrm{EtOH}} / V_{\text {water }}$ were investigated and summarized in Fig. S2A-B (ESI $\dagger)$. The small PILP volume is close to the detection limit of the AUC optics, and the data acquisition error is large. However, the PILP volume fraction is on the order of $10^{-4} \mathrm{vol} \%$, and does not increase noticeably in the existence range of PILPs. Therefore, in the present system, the amount of PILP phase is very small and cannot be increased. As a comparison, the volume fraction of coacervates can be as high as 0.7 (Fig. S3B, ESI $\dagger$ ). It increases linearly with [Glu] (or [PEI]) and decreases linearly with $V_{\text {EtOH }} / V_{\text {water }}$ (Fig. S3A-B, ESI $\dagger$ ).

In the next step, we investigated the composition of PILP as well as the supernatant. Considering the long induction time of precipitation via the PILP phase, the PILP phase was separated from the less dense supernatant phase by centrifugation. This shows that the PILP phase is richer in solute than the supernatant. The compositional analysis of both phases was performed by using ${ }^{1} \mathrm{H}$ NMR spectroscopy. The compositions of PILP and supernatant in two series $n_{\mathrm{Glu}} / n_{\mathrm{EI}}=1.5$ and $n_{\mathrm{Glu}} / n_{\mathrm{EI}}=0.9$ are shown in Fig. 4 . The key observation is that 


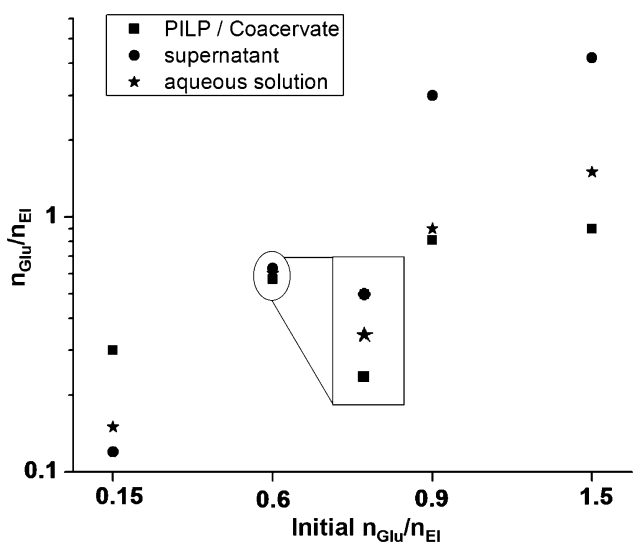

Fig. 4 The relationship between the values of $n_{\mathrm{Glu}} / n_{\mathrm{EI}}$ of the initial aqueous solution and the values of $n_{\mathrm{Glu}} / n_{\mathrm{EI}}$ from each phase after phase separation in the quaternary system $([\mathrm{Glu}]=0.12 \mathrm{~mol} \%$ in initial aqueous solution). The PILP phase was obtained when the values of $n_{\mathrm{Glu}} / n_{\mathrm{EI}}$ were at 0.9 and 1.5 ; while coacervation occurred when the values of $n_{\mathrm{Glu}} / n_{\mathrm{EI}}$ were at 0.6 and 0.15 . The data for $n_{\mathrm{Glu}} / n_{\mathrm{EI}}=0.6$ are magnified.

the $n_{\mathrm{Glu}} / n_{\mathrm{EI}}$ values in the PILP phases are lower than those in the supernatant; i.e., PILPs are richer in PEI compared to the supernatant phase. The higher PEI content decreases the precipitation driving force and stabilizes Glu for a few hours before it precipitates out. In addition, the compositional variation of the PILP phase is relatively small, as seen by measuring the values of $n_{\mathrm{Glu}} / n_{\mathrm{EI}}$ in different samples, compared to those of corresponding supernatant phases. Therefore, the compositional gap between the PILP phase and the supernatant increases with the increase of $n_{\mathrm{Glu}} / n_{\mathrm{EI}}$ from 0.9 to 1.5 , as shown in Fig. 4.

It is also of interest to detect the compositional change of the PILP phase with time. A series of PILP phases prepared by the same procedure and with the same composition were separated at various times. We found that the $n_{\mathrm{Glu}} / n_{\mathrm{EI}}$ value was constant with time from $1 \mathrm{~min}$ to $2 \mathrm{~h}$ (result not shown). This result suggests that there was no obvious compositional change in the PILP phase before the precipitation occurred. However, we cannot exclude the possibility that a subtle change of the composition of the PILP phase may induce the crystallization within the PILP phase and the crystallization proceeds by extruding polymers into the supernatant. This result is different from that from the $\mathrm{CaCO}_{3}$ inorganic PILP system, where it was observed that the polymeric additives were continuously expelled from the PILP with time. ${ }^{17}$

We also considered how parameters such as [Glu] $\left(n_{\mathrm{Glu}} / n_{\mathrm{EI}}\right.$ constant) and the $V_{\mathrm{EtOH}} / V_{\text {water }}$ value affect the compositions of the PILP as well as the supernatant phases (Fig. S4, ESI $\dagger$ ). NMR results show that the composition is nearly constant with either parameter. Therefore, the $n_{\mathrm{Glu}} / n_{\mathrm{EI}}$ value is the main parameter that determines the composition of both phases after phase separation.

The compositions of both phases from coacervation processes were measured as a comparison to the PILP systems. The results for $n_{\mathrm{Glu}} / n_{\mathrm{EI}}=0.6$ and $n_{\mathrm{Glu}} / n_{\mathrm{EI}}=0.15$ are also summarized in Fig. 4. For $n_{\mathrm{Glu}} / n_{\mathrm{EI}}=0.6$, the compositions of both phases formed are very close to the initial $n_{\mathrm{Glu}} / n_{\mathrm{EI}}$ value. The $n_{\mathrm{Glu}} / n_{\mathrm{EI}}$ value in the supernatant is still slightly higher than that in the dense phase. When the initial $n_{\mathrm{Glu}} / n_{\mathrm{EI}}$ value is lower than 0.6 , the $n_{\mathrm{Glu}} / n_{\mathrm{EI}}$ value in the coacervate becomes higher than that in the supernatant phase (see also Fig. S5, ESI $\dagger$ ). Between 0.05 and 0.04 in $n_{\mathrm{Glu}} / n_{\mathrm{EI}}$, the boundary of both phases disappears and homogeneous complexes are obtained thereafter.

Our results show that the compositional ratio between Glu and the polymeric additive PEI is a key parameter for the precipitation to proceed via the PILP route, which has been little explored so far. In our quaternary system, we detected two different possibilities for precipitation: precipitation from a clear solution and precipitation via the PILP route. Coacervates and homogeneous complexes were two stable phases which did not precipitate. Microspheres were formed via the PILP route (Fig. S1B and C, ESI †; crystalline microsphere superstructures with nanoplatelets as building blocks) and they are easily distinguishable from single crystalline precipitates obtained from a clear solution. In addition, the fast mixing process causes immediate formation of a large number density of PILP droplets. Importantly, the long induction period for crystallization from organic PILPs provides enough time for PILP separation and characterization.

To the best of our knowledge, the compositional investigation for crystallization via the PILP route is provided for the first time. In the current system, PILP is only obtainable in a narrow compositional range. More importantly, its amount is very low and was $\ll 1$ vol\% over the entire existence range of PILP. This suggests that PILPs cannot be upscaled in this system.

The PILP phase accumulates solute and is enriched in the polymer as compared to the initial solution. This explains the stabilization against crystallization for some time. However, in contrast to coacervates, with similar appearance, PILP always leads to crystals whereas coacervates remained stable. As a result of increased solute concentration the PILP phase has a higher density compared to the supernatant. The precipitation within each PILP droplet leads to microspheres with nanoplatelets as building blocks. The precipitation process will be discussed in a separate paper. ${ }^{37}$

\section{Conclusions}

The information about PILP formation herein can be conditionally transferred to other crystallization processes with polymeric additives, because different crystallization systems have distinct compositional ranges for the formation of the PILP phase. Crystallization via the PILP route can be a general phenomenon in crystallization with polymeric additives, although it can be difficult to detect due to the small existence region in the quaternary mixture. In addition, the difficulty to understand PILP formation is due to its fast transformation into crystalline structures in many systems. This difficulty can be solved by using those reaction model systems with constant composition and long induction time for crystallization, such as the precipitation of organics with polymeric additives. However, kinetic parameters such as mixing method and stirring are also influential factors in the formation of various far-from-equilibrium crystal morphologies via the PILP route, which makes the understanding of the PILP's role in crystallization with polymeric additives more difficult. 
The kinetic parameters for the preparation of crystals via the PILP route have been reported elsewhere. ${ }^{37}$ The information about the PILP formation determined in this work may be extrapolated to other crystallization processes with polymeric additives, leading to a new route for modulating crystal formation.

\section{Acknowledgements}

We acknowledge Prof. Dr Markus Antonietti for fruitful discussions. This work was supported by the DFG \& NSF "Materials World Network to Study Liquid Precursor Formation and Crystallization at Interfaces: Fundamentals towards Applications." YJ thanks The Max Planck Institute of Colloids and Interfaces for the financial support during 2008-2010. YJ acknowledges Antje Völker for analytical ultracentrifugation measurements, Olaf Niemeyer for NMR measurements, Ulrike Anders for handling parts of the pseudo ternary phase diagram determination experiments, and the electron microscopy group in MPIKG for microscopy support.

\section{References}

1 H. Cölfen and M. Antonietti, Mesocrystals and Nonclassical Crystallization, John Wiley \& Sons Ltd, 2008.

2 F. C. Meldrum and H. Cölfen, Chem. Rev., 2008, 108, 4332-4432.

3 J. Aizenberg, D. A. Muller, J. L. Grazul and D. R. Hamann, Science, 2003, 299, 1205-1208.

4 S. Weiner, I. Sagi and L. Addadi, Science, 2005, 309, 1027-1028.

5 R. L. Penn and J. F. Banfield, Science, 1998, 281, 969-971.

6 J. F. Banfield, S. A. Welch, H. Z. Zhang, T. T. Ebert and R. L. Penn, Science, 2000, 289, 751-754.

7 M. Niederberger and H. Cölfen, Phys. Chem. Chem. Phys., 2006, 8, 3271-3287.

8 P. R. ten Wolde and D. Frenkel, Science, 1997, 277, 1975-1978.

9 L. A. Gower and D. A. Tirrell, J. Cryst. Growth, 1998, 191, $153-160$.

10 L. B. Gower and D. J. Odom, J. Cryst. Growth, 2000, 210, 719-734.

11 L. B. Gower, Chem. Rev., 2008, 108, 4551-4627.

12 S. Wohlrab, H. Cölfen and M. Antonietti, Angew. Chem., Int. Ed., 2005, 44, 4087-4092.
13 O. Galkin and P. G. Vekilov, Proc. Natl. Acad. Sci. U. S. A., 2000, 97, 6277-6281.

14 L. Addadi, S. Raz and S. Weiner, Adv. Mater., 2003, 15, 959-970.

15 Y. Politi, T. Arad, E. Klein, S. Weiner and L. Addadi, Science, 2004, 306, 1161-1164.

16 P. G. Vekilov, Cryst. Growth Des., 2004, 4, 671-685.

17 L. Dai, E. P. Douglas and L. B. Gower, J. Non-Cryst. Solids, 2008, 354, 1845-1854.

18 N. Gehrke, N. Nassif, N. Pinna, M. Antonietti, H. S. Gupta and H. Cölfen, Chem. Mater., 2005, 17, 6514-6516.

19 D. Volkmer, M. Harms, L. Gower and A. Ziegler, Angew. Chem., Int. Ed., 2005, 44, 639-644.

20 G. Falini, S. Albeck, S. Weiner and L. Addadi, Science, 1996, 271, 67-69.

21 M. J. Olszta, E. P. Douglas and L. B. Gower, Calcif. Tissue Int., 2003, 72, 583-591.

22 M. J. Olszta, X. G. Cheng, S. S. Jee, R. Kumar, Y. Y. Kim, M. J. Kaufman, E. P. Douglas and L. B. Gower, Mater. Sci. Eng., $R, 2007, \mathbf{5 8}, 77-116$.

23 S. S. Jee, T. T. Thula and L. B. Gower, Acta Biomater., 2010, 6, $3676-3686$.

24 D. Gebauer, A. Völkel and H. Cölfen, Science, 2008, 322 1819-1822.

25 E. M. Pouget, P. H. H. Bomans, J. Goos, P. M. Frederik, G. de With and N. Sommerdijk, Science, 2009, 323, 1455-1458.

26 F. Nudelman, K. Pieterse, A. George, P. H. H. Bomans, H. Friedrich, L. J. Brylka, P. A. J. Hilbers, G. de With and N. A. J. M. Sommerdijk, Nat. Mater., 2010, 9, 1004-1009.

27 S. J. Homeijer, R. A. Barrett and L. B. Gower, Cryst. Growth Des., 2010, 10, 1040-1052.

28 S. J. Homeijer, M. J. Olszta, R. A. Barrett and L. B. Gower, J. Cryst. Growth, 2008, 310, 2938-2945.

29 Y. Y. Kim, E. P. Douglas and L. B. Gower, Langmuir, 2007, 23, $4862-4870$.

30 X. G. Cheng and L. B. Gower, Biotechnol. Prog., 2006, 22, 141-149.

31 F. F. Amos, D. M. Sharbaugh, D. R. Talham, L. B. Gower, M. Fricke and D. Volkmer, Langmuir, 2007, 23, 1988-1994.

32 E. DiMasi, S. Y. Kwak, F. F. Amos, M. J. Olszta, D. Lush and L. B. Gower, Phys. Rev. Lett., 2006, 97, 045503.

33 E. DiMasi, V. M. Patel, M. Sivakumar, M. J. Olszta, Y. P. Yang and L. B. Gower, Langmuir, 2002, 18, 8902-8909.

34 Y. R. Ma, G. Mehltretter, C. Plug, N. Rademacher, M. U. Schmidt and H. Cölfen, Adv. Funct. Mater., 2009, 19, 2095-2101.

35 V. A. Pertzoff, J. Biol. Chem., 1933, 100, 97.

36 M. S. Dunn and M. P. Stoddard, J. Biol. Chem., 1937, 121, 521-529.

37 Y. Jiang, L. B. Gower, D. Volkmer and H. Cölfen, Cryst. Growth Des., 2011, 11, 3243-3249. 